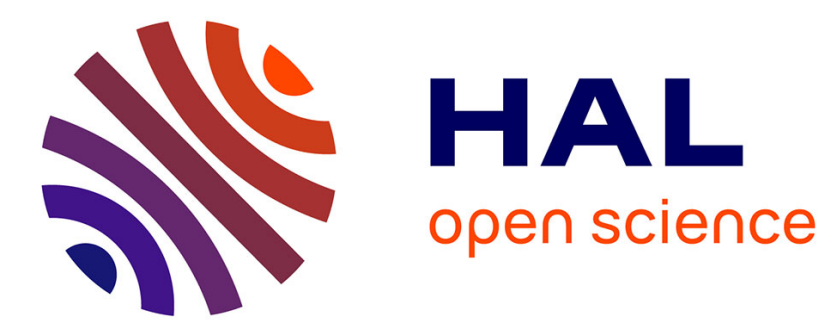

\title{
Stockage thermique en milieu poreux granulaire
}

\author{
A. Boisdet, J.L. Peube, D. Blay
}

\section{To cite this version:}

A. Boisdet, J.L. Peube, D. Blay. Stockage thermique en milieu poreux granulaire. Revue de Physique Appliquée, 1982, 17 (9), pp.591-594. 10.1051/rphysap:01982001709059100 jpa-00245036

\section{HAL Id: jpa-00245036 https://hal.science/jpa-00245036}

Submitted on 1 Jan 1982

HAL is a multi-disciplinary open access archive for the deposit and dissemination of scientific research documents, whether they are published or not. The documents may come from teaching and research institutions in France or abroad, or from public or private research centers.
L'archive ouverte pluridisciplinaire HAL, est destinée au dépôt et à la diffusion de documents scientifiques de niveau recherche, publiés ou non, émanant des établissements d'enseignement et de recherche français ou étrangers, des laboratoires publics ou privés. 


\title{
Stockage thermique en milieu poreux granulaire
}

\author{
A. Boisdet, J. L. Peube et D. Blay \\ Laboratoire d'Energétique Solaire, 40, avenue du Recteur Pineau, 86022 Poitiers cedex, France
}

(Reçu le 17 septembre 1981, révisé le 10 février 1982, accepté le 15 février 1982)

\begin{abstract}
Résumé. - On étudie le comportement thermique d'un système de stockage en milieu poreux granulaire. A partir d'un modèle mathématique unidimensionnel, on détermine les champs de température du milieu solide et du fluide au cours des phases d'évolution dynamique. On évalue l'ordre de grandeur de l'épaisseur du front thermique caractéristique de l'état de stratification du stock. Les résultats numériques sont ensuite confrontés, dans le cas d'une phase de charge, aux résultats expérimentaux obtenus dans un stockage de billes d'alumine utilisant l'air à $300^{\circ} \mathrm{C}$ environ comme fluide caloporteur. Les écarts obtenus sont inférieurs à $10 \%$.
\end{abstract}

\begin{abstract}
The thermal behaviour of a packed-bed storage system is studied. Using a one-dimensional mathematical model, solid and fluid temperatures are determined during dynamic evolution phases. The order of magnitude of the thermocline thickness, characteristic of the thermal stratification is evaluated. Then, numerical results are compared, for a loading phase, to experimental results obtained in the case of a storage using alumina spheres and air as thermal fluid. Discrepancies are less than $10 \%$.
\end{abstract}

\section{Nomenclature.}

$a \quad:$ Diffusivité thermique $\left(\mathrm{m}^{2} / \mathrm{s}\right)$.

$d$ : Dimension caractéristique d'une particule $(\mathrm{m})$.

$l$ : Epaisseur du front thermique (m).

$l^{*}:$ Epaisseur adimensionnelle du front thermique.

$L$ : Hauteur de l'accumulateur (m).

$N u:$ Nombre de Nusselt $=\frac{\alpha L}{\lambda_{\mathrm{f}}}$.

$\operatorname{Pr}:$ Nombre de Prandtl $=\frac{v_{\mathrm{f}}}{a_{\mathrm{f}}}$.

$R e:$ Nombre de Reynolds $=\frac{u \cdot d}{v_{\mathrm{f}}}$.

St $:$ Nombre de Stanton $=N u / R e . P r$.

$t$ : Temps (s).

$t_{0}:$ Temps caractéristique (s).

$T$ : Température $\left({ }^{\circ} \mathrm{C}\right)$.

$T_{\mathrm{e}}$ : Température d'entrée du fluide $\left({ }^{\circ} \mathrm{C}\right)$.

$u$ : Vitesse réelle du fluide $(\mathrm{m} / \mathrm{s})$.

$U$ : Vitesse adimensionnelle du fluide $=u t_{0} / L$.

$u^{*}$ : Vitesse de débit du fluide rapportée à la section de l'accumulateur $(\mathrm{m} / \mathrm{s})$.

$v$ : Vitesse de déplacement du front thermique $(\mathrm{m} / \mathrm{s})$.

$v_{\mathrm{f}}:$ Vitesse relative du fluide $(\mathrm{m} / \mathrm{s})$.

$v_{\mathrm{s}}:$ Vitesse relative du solide $(\mathrm{m} / \mathrm{s})$.

$x$ : Cote $(\mathrm{m})$.

$X$ : Cote adimensionnelle.
Symboles grecs.

$\alpha$ : Coefficient d'échange thermique $\left(\mathrm{W} / \mathrm{m}^{2}{ }^{\circ} \mathrm{C}\right)$.

$\varepsilon$ : Porosité du milieu granulaire.

$\theta:$ Température adimensionnelle.

$\xi$ : Cote adimensionnelle.

$\rho$ : Masse volumique $\left(\mathrm{kg} / \mathrm{m}^{3}\right)$.

$\Sigma$ : Taux de surface de contact solide-fluide $\left(\mathrm{m}^{-1}\right)$

$\tau$ : Temps adimensionnel.

\section{Indices.}

f : se rapportant au fluide.

$s$ : se rapportant au solide.

0 : se rapportant à l'instant initial.

Introduction. - Le transfert de chaleur entre un fluide et un milieu poreux a un intérêt important dans de nombreux processus industriels tels que les régénérateurs, les réacteurs catalytiques et nucléaires et les unités de stockage thermique. Une application récente concerne plus particulièrement le stockage de la chaleur, à basse ou haute température, provenant de collecteurs solaires.

De nombreux travaux ont été consacrés à ce problème. Ces études sont essentiellement théoriques, elles proposent des solutions analytiques ou numériques [1] à [5] et analysent la réponse dynamique du système pour différents types de conditions aux 
limites et initiales. Dans tous les cas, les modèles mathématiques présentés se prêtent mal à des simulations sur de longues durées et ne sont donc pas adaptés au problème du stockage thermique de grande capacité.

L'originalité du travail présenté ici est d'apporter une contribution ayant principalement pour but de fixer certains ordres de grandeur caractéristiques relatifs au front thermique qui se développe lors des phases de charge et de décharge de l'accumulateur. Sa finalité est donc essentiellement pratique et orientée vers le dimensionnement de systèmes de stockage réels.

1. Aspects théoriques. - 1.1 Equations. - En considérant le problème unidimensionnel selon l'axe parallèle à la vitesse de l'écoulement fluide, les équations qui définissent le champ instationnaire de température du fluide et du milieu poreux s'écrivent, dans un système de coordonnées fixe :

$$
\begin{aligned}
\rho_{\mathrm{f}} C_{\mathrm{f}} \varepsilon \frac{\partial T_{\mathrm{f}}}{\partial t}+u \frac{\partial T_{\mathrm{f}}}{\partial x} & =\alpha \Sigma\left(T_{\mathrm{s}}-T_{\mathrm{f}}\right) \\
\rho_{\mathrm{s}} C_{\mathrm{s}}(1-\varepsilon) \frac{\partial T_{\mathrm{s}}}{\partial t} & =\alpha \Sigma\left(T_{\mathrm{f}}-T_{\mathrm{s}}\right) .
\end{aligned}
$$

Ces équations sont obtenues à partir des hypothèses suivantes :

- la section de l'accumulateur est constante,

- les paramètres physiques ne varient pas avec la température,

- les transferts par conduction axiale et rayonnement sont négligés,

- les particules constituant le milieu poreux sont supposées isothermes (le nombre de Biot caractérisant l'échange est petit devant 1 ),

- le système est adiabatique.

Le système d'équations (1) et (2) est complété par les conditions aux limites et initiales suivantes :

$$
\begin{aligned}
& T_{\mathrm{f}}(0, t)=T_{\mathrm{e}}=\text { Cte } \\
& T_{\mathrm{f}}(x, 0)=T_{\mathrm{s}}(x, 0)=T_{0}=\text { Cte } .
\end{aligned}
$$

Si l'on pose

$$
\begin{aligned}
\tau & =\frac{t}{t_{0}} & X & =\frac{x}{L} \\
\theta_{\mathrm{f}} & =\frac{T_{\mathrm{f}}-T_{0}}{T_{\mathrm{e}}-T_{0}}, & \theta_{\mathrm{s}} & =\frac{T_{\mathrm{s}}-T_{0}}{T_{\mathrm{e}}-T_{0}}
\end{aligned}
$$

où $t_{0}=\frac{(1-\varepsilon) \rho_{\mathrm{s}} C_{\mathrm{s}} L}{\varepsilon \rho_{\mathrm{f}} C_{\mathrm{f}} u^{*}}$ est un temps caractéristique du sțockage, les équations (1) à (4) deviennent

$$
\begin{aligned}
\frac{\partial \theta_{\mathrm{f}}}{\partial \tau}+U \frac{\partial \theta_{\mathrm{f}}}{\partial X} & =A\left(\theta_{\mathrm{s}}-\theta_{\mathrm{f}}\right) \\
\frac{\partial \theta_{\mathrm{s}}}{\partial \tau} & =B\left(\theta_{\mathrm{f}}-\theta_{\mathrm{s}}\right)
\end{aligned}
$$

avec

$$
\begin{aligned}
A & =\frac{L \Sigma S t}{\varepsilon} \\
B & =\frac{L \Sigma S t}{(1-\varepsilon)} \frac{\rho_{\mathrm{f}} C_{\mathrm{f}}}{\rho_{\mathrm{s}} C_{\mathrm{s}}} \\
S t & =\frac{N u}{\operatorname{RePr}} \\
U & =\frac{u t_{0}}{L} \\
\theta_{\mathbf{f}}(0, \tau) & =1 \\
\theta_{\mathrm{f}}(X, 0) & =\theta_{\mathrm{s}}(X, 0)=0 .
\end{aligned}
$$

1.2 RéSOLUTION NUMÉRIQUE. - Les équations (5) à (8) possèdent une solution analytique sous forme de développements en séries [1], [5]. Elle présente cependant l'inconvénient d'être limitée à des systèmes dont le temps caractéristique $t_{0}$ est faible (de l'ordre de la minute).

L'application au stockage thermique met en jeu des temps caractéristiques beaucoup plus grands. Aussi ces équations ont-elles été résolues à partir d'un schéma explicite aux différences finies adapté à leur nature hyperbolique.

La discrétisation des équations à partir d'un maillage spatial et temporel de pas respectivement égaux à $U k$ et $k$, perrmet d'écrire :

La discrétisation des équations à partir d'un maillage spatial et temporel de pas respectivement égaux à $U k$ et $k$, permet d'écrire :

$$
\begin{aligned}
& \theta_{f_{i}}^{j+1}=\theta_{f_{i-1}}^{j}+A k\left(\theta_{s_{i-1}}^{j}-\theta_{f_{i-1}}^{j}\right) \\
& \theta_{s_{i}}^{j+1}=\theta_{s_{i-1}}^{j}+B k\left(\theta_{f_{i-1}}^{j}-\theta_{s_{i-1}}^{j}\right) \\
& \theta_{f_{0}}^{j}=1 \\
& \theta_{f_{i}}^{0}=\theta_{s_{i}}^{0}=0
\end{aligned}
$$

où

$$
\begin{array}{ll}
0 \leqslant X \leqslant m U k, & i=0,1, \ldots m \\
0 \leqslant \tau \leqslant n k, & j=0,1, \ldots n
\end{array}
$$

La figure 1 montre l'évolution des températures du fluide et des particules solides au cours d'une phase de stockage et celle du front thermique qui s'est formé. On constate que celui-ci s'épaissit au cours de la charge affectant ainsi la qualité de la stratification.

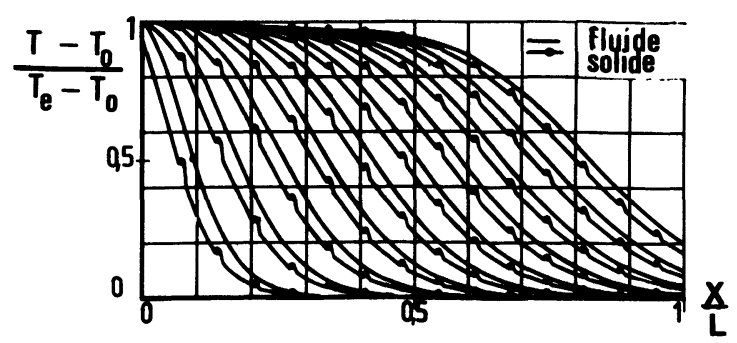

Fig. 1. - Evolution de la température du fluide et du milieu solide durant une phase de charge $\Delta \tau=0,123$.

[Fluid and solid temperature evolution during a loading phase $\Delta \tau=0.123$.] 
1.3 Analyse Qualitative. - Cette analyse a pour but de donner des ordres de grandeur des paramètres caractéristiques du front thermique : épaisseur, vitesse de déplacement, écart de température entre le fluide et le solide.

Si l'on considère que ce front garde la même allure durant la phase d'évolution, il est possible de définir une vitesse équivalente de sa propagation comme étant la vitesse, $v$, de déplacement de son point moyen (point d'inflexion du profil). En d'autres termes, on définit ainsi cette vitesse de propagation comme celle d'un système de coordonnées mobile dans lequel le flux thermique est globalement nul.

Dans ce nouveau système de coordonnées défini à partir du changement de variables :

$$
\xi=x-v t
$$

les équations (1) et (2) s'écrivent :

$$
\begin{array}{r}
\rho_{\mathrm{f}} C_{\mathrm{f}} \varepsilon\left[\frac{\partial T_{\mathrm{f}}}{\partial t}+v_{\mathrm{f}} \frac{\partial T_{\mathrm{f}}}{\partial \xi}\right]=\alpha \Sigma\left(T_{\mathrm{s}}-T_{\mathrm{f}}\right) \\
\rho_{\mathrm{s}} C_{\mathrm{s}}(1-\varepsilon)\left[\frac{\partial T_{\mathrm{s}}}{\partial t}+v_{\mathrm{s}} \frac{\partial T_{\mathrm{s}}}{\partial \xi}\right]=\alpha \Sigma\left(T_{\mathrm{f}}-T_{\mathrm{s}}\right)
\end{array}
$$

où

$$
\begin{aligned}
& v_{\mathrm{f}}=u-v \\
& v_{\mathrm{s}}=-v .
\end{aligned}
$$

En ajoutant les équations (9) et (10), il vient :

$$
\begin{aligned}
\rho_{\mathrm{f}} C_{\mathrm{f}} \varepsilon\left[\frac{\partial T_{\mathrm{f}}}{\partial t}+v_{\mathrm{f}} \frac{\partial T_{\mathrm{f}}}{\partial \xi}\right]+ \\
\quad+\rho_{\mathrm{s}} C_{\mathrm{s}}(1-\varepsilon)\left[\frac{\partial T_{\mathrm{s}}}{\partial t}+v_{\mathrm{s}} \frac{\partial T_{\mathrm{s}}}{\partial \xi}\right]=0 .
\end{aligned}
$$

Après intégration de (11) sur l'intervalle $\left[\xi_{1}, \xi_{2}\right]$, du front thermique où $\xi_{1}$ et $\xi_{2}$ sont définies de façon que

$$
\begin{aligned}
& T_{\mathrm{f}}\left(\xi_{1}, t\right)=T_{\mathrm{s}}\left(\xi_{1}, t\right)=T_{\mathrm{e}} \\
& T_{\mathrm{f}}\left(\xi_{2}, t\right)=T_{\mathrm{s}}\left(\xi_{2}, t\right)=T_{0}
\end{aligned}
$$

On obtient :

$$
\begin{aligned}
& \frac{\partial}{\partial t}\left[\int_{\xi_{2}}^{\xi_{1}}\left(\rho_{\mathrm{f}} C_{\mathrm{f}} \varepsilon T_{\mathrm{f}}+\rho_{\mathrm{s}} C_{\mathrm{s}}(1-\varepsilon) T_{\mathrm{s}}\right) \mathrm{d} \xi\right]+ \\
& \quad+\rho_{\mathrm{f}} C_{\mathrm{f}} \varepsilon v_{\mathrm{f}}\left[T_{\mathrm{e}}-T_{\mathrm{o}}\right]+\rho_{\mathrm{s}} C_{\mathrm{s}}(1-\varepsilon) v_{\mathrm{s}}\left[T_{\mathrm{e}}-T_{0}\right]=0
\end{aligned}
$$

Dans le système de coordonnées $(\xi, t)$, le phénomène peut être considéré comme stationnaire. Il s'ensuit donc que le premier terme de cette équation est nul et par conséquent :

$$
\rho_{\mathrm{f}} C_{\mathrm{f}} \varepsilon v_{\mathrm{f}}\left[T_{\mathrm{e}}-T_{\mathrm{o}}\right]+\rho_{\mathrm{s}} C_{\mathrm{s}}(1-\varepsilon) v_{\mathrm{s}}\left[T_{\mathrm{e}}-T_{\mathrm{o}}\right]=0 .
$$

D'où :

$$
v=\frac{\rho_{\mathrm{f}} C_{\mathrm{f}} \varepsilon u}{\rho_{\mathrm{f}} C_{\mathrm{f}} \varepsilon+\rho_{\mathrm{s}} C_{\mathrm{s}}(1-\varepsilon)} .
$$

En déterminant l'ordre de grandeur des termes de l'équation (11) et compte tenu de (12), on obtient l'ordre de grandeur de l'épaisseur du front thermique, $l=\left|\xi_{1}-\xi_{2}\right|$ :

$$
l \simeq \frac{v_{\mathrm{f}}}{u}\left[v t \frac{\varepsilon}{\Sigma S t}\right]^{1 / 2}
$$

$l$ croît proportionnellement à $t^{1 / 2}$. Si l'on définit l'épaisseur relative, $l^{*}$, du front thermique en posant :

$$
l^{*}=\frac{l}{v t} \text {. }
$$

Il vient :

$$
l^{*} \simeq \frac{v_{\mathrm{f}}}{u}\left[\frac{\varepsilon}{v t \Sigma S t}\right]^{1 / 2} .
$$

Par ailleurs, l'intégration de l'équation (9) sur l'intervalle $\left[\xi_{1}, \xi_{2}\right]$ donne :

$$
\begin{aligned}
\rho_{\mathrm{f}} C_{\mathrm{f}} \varepsilon \int_{\xi_{2}}^{\xi_{1}} \frac{\partial T_{\mathrm{f}}}{\partial t} \mathrm{~d} \xi+\rho_{\mathrm{f}} C_{\mathrm{f}} \varepsilon v_{\mathrm{f}}\left[T_{\mathrm{e}}-T_{0}\right] & \\
& =\alpha \Sigma \int_{\xi_{2}}^{\xi_{1}}\left(T_{\mathrm{s}}-T_{\mathrm{f}}\right) \mathrm{d} \xi .
\end{aligned}
$$

A partir du calcul d'ordre de grandeur des différents termes, on obtient l'ordre de grandeur de la différence de température $\left(T_{\mathrm{f}}-T_{\mathrm{s}}\right)$ :

$$
\left(T_{\mathrm{f}}-T_{\mathrm{s}}\right) \simeq \frac{\rho_{\mathrm{f}} C_{\mathrm{f}} v_{\mathrm{f}} \varepsilon\left(T_{\mathrm{e}}-T_{0}\right)}{\alpha \Sigma l}
$$

c'est-à-dire compte tenu de (13)

$$
\left(T_{\mathrm{f}}-T_{\mathrm{s}}\right) \simeq \frac{\rho_{\mathrm{f}} C_{\mathrm{f}} u\left(T_{\mathrm{e}}-T_{0}\right)}{\alpha}\left\lceil\left.\frac{\varepsilon S t}{v t \Sigma}\right|^{1 / 2} .\right.
$$

Ainsi, la différence de température $\left(T_{\mathrm{f}}-T_{\mathrm{s}}\right)$, et l'épaisseur relative du front thermique, $l^{*}$, sont proportionnelles à $t^{-1 / 2}$ ce qui signifie que la stratification thermique sera d'autant meilleure que l'accumulateur sera défini par un temps caractéristique plus élevé, autrement dit, que l'accumulateur est de grande capacité.

2. Aspects expérimentaux. -2.1 DESCRIPTION DU MONTAGE EXPÉRIMENTAL. - Afin de vérifier la validité des résultats théoriques obtenus, une boucle de stockage, de dimensions comparables à celles d'un stockage solaire diurne, a été réalisée [7]. Elle est principalement constituée d'un container cylindrique d'axe vertical, isolé thermiquement, de $2 \mathrm{~m}$ de hauteur et $0,54 \mathrm{~m}$ de diamètre, rempli de $875 \mathrm{~kg}$ de billes sphériques d'alumine de $2,5 \mathrm{~cm}$ de diamètre. Le fluide thermique utilisé est de l'air préchauffé sur des résistances électriques d'une puissance de $30 \mathrm{~kW}$, à une température pouvant atteindre $400^{\circ} \mathrm{C}$ (Fig. 2). 


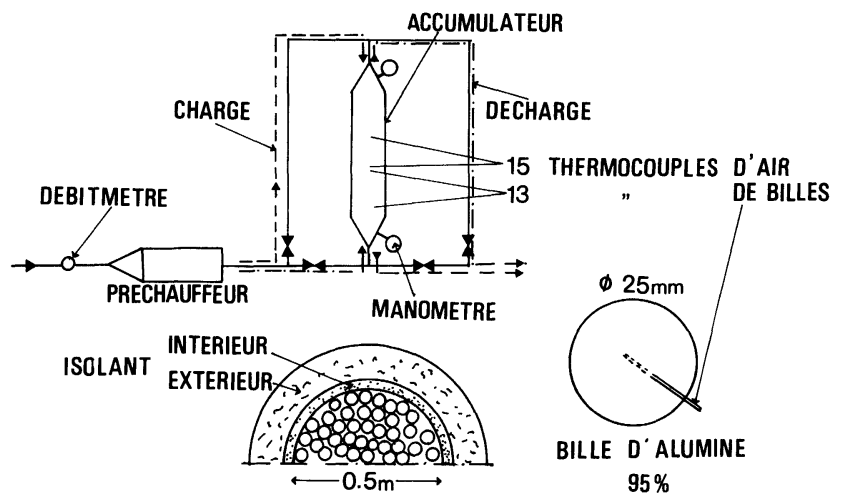

Fig. 2. - Schéma de l'installation expérimentale.

[Test apparatus diagram.]

Les températures sont mesurées à l'aide de 28 thermocouples, dont 15 répartis dans l'air et 13 à l'intérieur des billes.

L'écoulement d'air est assuré par un compresseur à débit constant, d'environ $90 \mathrm{~m}^{3} / \mathrm{h}$, mesuré à partir d'un débitmètre à flotteur disposé en amont du préchauffeur.

Les résultats expérimentaux ont permis d'abord

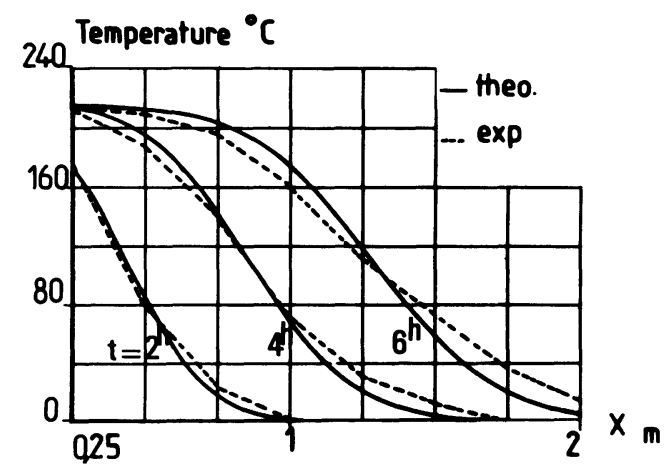

Fig. 3. - Comparaison des résultats numériques et expérimentaux lors d'une phase de charge.

[Numerical and experimental temperatures during a loading phase.] de vérifier la validité de certaines hypothèses utilisées pour l'établissement du modèle mathématique :

- l'isothermicité d'une bille

- les faibles variations de température dans une section droite du cylindre. Ces variations sont provoquées par une non-homogénéité de la porosité près des parois qui crée une zone d'écoulement privilégiée. L'écart de température relatif maximum ne dépasse pas $10 \%$.

Sur la figure 3 , on montre l'évolution des champs de température du milieu solide et du fluide au cours d'une phase de charge caractérisée par les conditions suivantes :

$$
\begin{aligned}
\varepsilon & =0,40 \\
u^{*} & =0,1 \mathrm{~m} / \mathrm{s} \\
T_{\mathrm{e}} & =220^{\circ} \mathrm{C} \\
T_{0} & =0{ }^{\circ} \mathrm{C} \\
\Sigma & =144 \mathrm{~m}^{-1} .
\end{aligned}
$$

Les valeurs expérimentales y sont comparées aux valeurs numériques obtenues à partir de la résolution des équations (1) à (4) où le coefficient d'échange de chaleur, $\alpha$, a été déterminé à partir des relations suivantes [6] :

$$
\begin{aligned}
& R e<600 \frac{\varepsilon^{3}}{1-\varepsilon} \\
& N u=2,62 \times 10^{-4}\left[\frac{(1-\varepsilon)}{\varepsilon^{3}} \operatorname{Re}\right]^{1,6} \\
& \operatorname{Re}>600 \frac{\varepsilon^{3}}{1-\varepsilon} \\
& N u=6,6 \times 10^{-2}\left[\frac{(1-\varepsilon)}{\varepsilon^{3}} \operatorname{Re}\right]^{0,75} .
\end{aligned}
$$

Les résultats obtenus confirment la validité du modèle et des ordres de grandeurs. En effet, les écarts qui subsistent et qui sont essentiellement dus à la conduction axiale et au transfert radiatif négligés dans l'étude théorique restent faibles puisque l'écart relatif maximum au bout d'une durée de charge de 6 heures est de $7 \%$.

\section{Bibliographie}

[1] Oiknine, C., Robert, C., Transfert de chaleur entre un fluide incompressible et un milieu poreux. Rev. Gen. Therm. VII (1969) 151-164.

[2] Grangeot, G., Etude théorique de l'écoulement et du transfert de chaleur entre un fluide compressible et un milieu poreux. Rev. Gen. Therm., $\mathrm{n}^{0}$ 186-187 (1977) 509-515.

[3] JANG, W. J., LEE, C. P., Dynamic Response of Solar Heat Storage Systems, ASME Paper no 74-WA/HT 22 (1974).

[4] Hughes, P. J., Klein, S. A., Close, D. J., Packed bed thermal storage models for solar air heating and cooling systems. J. Heat Trans. (1976) p. 336-337.
[5] RiAz, M., Transient Analysis of Packed Bed Thermal Storage Systems. Comptes-Rendus de l'I.S.E.C. Atlanta (U.S.A.) juin 79.

[6] Donnadieu, G., Transmission de la chaleur entre fluide et solide. Revue de l'Institut Français du Pétrole, XVI (1961) p. 1330-1356.

[7] Boisdet, A., Contribution à l'étude du stockage thermique en milieu poreux granulaire. Thèse de Docteur-Ingénieur Poitiers (déc. 78).

[8] Boisdet, A., Peube, J. L., Thermal storage in Rock-beds. I.S.E.C. Proceedings Atlanta (U.S.A.) juin 79. 